\title{
Crashworthiness Determination for Front and Rear Doors and B Pillar Subjected to Side Impact Crash by a Mobile Deformable Barrier.
}

\begin{abstract}
In Euro NCAP standard, adult protection is one of the most important rating scores with 50\% weight factor while child protection and pedestrian protection are accounted into consideration with $20 \%$ weight factor. For adult protection testing, three tests are required to perform: (1) side impact, (2) pole impact, (3) front impact. In the side impact test, dummy's head, chest, shoulder, thorax, ribs, abdomen, pelvic and femur must be studied to evaluate the rating score. Crashworthiness of a car during side impact can describe the score rated for that car. In this paper the goal is to determine the crashworthiness of side doors and B-pillar in side impact crash test by simulation using LS DYNA software in order to predict required changes to achieve 5 star rating. A model of car has been developed using Hypermesh and CATIA software. A mobile deformable barrier (MDB) model and its honey comp area have been prepared with respect to ECE R95 and Euro NCAP standard. Initial condition such as velocity has been assigned to MDB. Results show that improvement in design in order to increase absorbed energy can probably has positive effect on crash performance as good as using side air bag in order to achieve 5 star rating.
\end{abstract}

Keyword: Crashworthiness; Side impact; Crash simulation; NCAP 